of CRC in the two groups. Chi-squared testing was used to assess for differences in the proportion of CRC between MMR groups with $\mathrm{p}<0.05$ considered significant.

Results Among 988 patients with EC not associated with a germline MMR mutation, 16\% $(\mathrm{n}=162)$ had MLH-1 promotor hypermethylation and $84 \%(n=826)$ did not. Among those with MLH-1 promotor hypermethylation there were 6 cases (3.6\%) of CRC vs. 34 cases $(4.1 \%)$ in those with MSS disease $(\mathrm{p}=.743)$.

Conclusions We found no difference in incidence of CRC in individuals with MLH-1 promotor hypermethylated EC as compared with those with MSS disease. Patients with MLH-1 promotor hypermethylated EC should follow general CRC screening guidelines.

\section{EPV096/\#110 LSR ACTIVATES MAPK PATHWAY AND PROMOTES CELL PROLIFERATION AND INVASION IN ENDOMETRIAL CANCER: ANALYSIS OF BIOINFORMATICS-BASED SIGNAL TRANSDUCTION}

${ }^{1} Y$ Nagase*, ${ }^{1} \mathrm{~K}$ Hiramatsu, 'S Nakagawa, 'S Matsuzaki, 'T Kimura, ${ }^{2} \mathrm{~S}$ Serada, ${ }^{1} Y$ Ueda, ${ }^{2} \mathrm{~T}$ Naka, ${ }^{1} \mathrm{~T}$ Kimura. ${ }^{1}$ Osaka University Graduate School of Medicine, Department of Obstetrics and Gynecology, Suita, Osaka, Japan; ${ }^{2}$ Kochi University, Department of Clinical Immunology, Nankoku, Kochi, Japan

\subsection{6/ijgc-2021-IGCS.166}

Objectives Lipolysis-stimulated lipoprotein receptor (LSR) is a membrane protein that has been studied in various malignant tumors. We previously reported that high expression of LSR was associated with poor prognosis, advanced stage, deep myometrial invasion, and metastasis in endometrial cancer (EC). However, the mechanism by which LSR affects patient's prognosis remains largely unclear. Here, we aimed to investigate the functions of LSR in EC.

Methods Cell proliferation and invasion were analyzed using LSR-knockdown cell lines (HEC1 and HEC116), and the activity of several signaling pathways were examined by Western blotting. To investigate the function of LSR in EC cells, the pathway enrichment and ontology analysis were performed using the publicly available proteomic data.

Results LSR-knockdown significantly suppressed cell proliferation in WST-8 assay. The pathway analysis demonstrated that MAPK signaling pathway was enriched in proteins correlated with high LSR expression. In ontology analysis, we found several biological processes, including 'regulation of ERK1/2' and 'MAPK cascade.' Following the results of pathway enrichment and ontology analysis, we confirmed that LSR-knockdown downregulated the phosphorylation of MEK/ERK pathway, including MEK1/2, ERK1/2, and p90RSK in western blotting. Cell invasion assay and western blot analysis demonstrated that LSR-knockdown suppressed MT1-MMP/MMP2 expression and cell invasion. Interestingly, ERK1/2-knockdown also suppressed MT1-MMP/MMP2 expression, suggesting that LSR activated MT1-MMP/MMP2 via ERK1/2 and promoted cell invasion.

Conclusions Our results of in vitro study and bioinformatic analysis showed that LSR regulated cell proliferation and invasion via $\mathrm{MEK} / \mathrm{ERK}$ pathway, and contributed poor prognosis in EC. LSR may be a new therapeutic target of advanced EC.

\section{EPV097/\#140 APPLICATION OF A MACHINE LEARNING ALGORITHM TO IDENTIFY PREDICTORS OF RECURRENCE AND RECURRENCE FREE SURVIVAL IN HIGH GRADE ENDOMETRIAL CANCER}

${ }^{1} \mathrm{~S}$ Piedimonte*, ${ }^{2} \mathrm{~T}$ Feigenberg, ${ }^{3} \mathrm{~B}$ Cormier, ${ }^{4} \mathrm{~J}$ Kwon, ${ }^{5} \mathrm{~W}$ Gotlieb, ${ }^{6} \mathrm{M}$ Plante, ${ }^{5} \mathrm{~S}$ Lau ${ }^{7} \mathrm{~L}$ Helpman, ${ }^{6} \mathrm{MC}$ Renaud, ${ }^{8} \mathrm{~T}$ May, ${ }^{9} \mathrm{D}$ Vicus. ' University of Toronto, Gynecologic Oncolgoy, Toronto, Canada; ${ }^{2}$ Trillium Health Partners, Gynecologic Oncolgoy, missassauga, Canada; ${ }^{3}$ Centre hospitalier de I'Université de Montréal, Gynecologic Oncology, Montreal, Canada; ${ }^{4}$ Vancouver General Hospital, Gynecologic Oncolgoy, Vancouver, Canada; ${ }^{5}$ McGill University, Jewish General Hospital, Gynecology Oncology, Montreal, Canada; ${ }^{6}$ Hotel Dieu de Quebec, Gynecology Oncology, Quebec, Canada; ${ }^{7} J u r a v i n s k i$ Cancer Center, Gynecologic Oncology, Hamilton, Canada; ${ }^{8}$ Princess Margaret Cancer Centre/University of Health Network/Sinai Health Systems, Gynecologic Oncology, Toronto, Canada; ${ }^{9}$ Sunnybrook Health Sciences Centre, Gynecologic Oncology, Toronto, Canada

\subsection{6/ijgc-2021-IGCS.167}

Objectives To train various machine learning algorithms to predict recurrence and recurrence-free survival (RFS) in highgrade endometrial cancer (HGEC)

Methods Data was retrospectively collected across 8 Canadian centers including 1237 patients and divided arbitrarily 50\% training, 25\% validation and 25\% testing. Four models were trained to predict recurrence: random forests, boosted trees, and 2 neural networks. Receiver operating characteristic curves (ROC) were used to determine model performance and select the best model based on highest area under the curve (AUC) in the test set. For time to recurrence models, we trained a random forest and Lasso model compared to Cox Proportional hazards. Concordance was reported using a c-statistic.

Results Among the 4 models tested, the bootstrap random forest had the best AUC in the test set and was the best model to predict recurrence in HGEC; the AUCs were $85.2 \%, 74.1 \%$ and $71.8 \%$ in the training, validation and test sets respectively. The top 5 predictors were: stage, uterus height, specimen weight, adjuvant chemotherapy and pre-operative histology. When stratified by stage, the AUC in the test set increased to $77 \%$ for Stage III and $80 \%$ for Stage IV. For time to recurrence, there was no difference between the Lasso and Cox Proportional Hazards models (test set c-index 71\%) while the random forest had a cindex of $60.5 \%$.

Conclusions A bootstrap random forest model best predicted recurrence in HGEC; model prediction further improved in Stage III and IV patients. Machine learning survival models performed similar to Cox Proportional Hazards but could be conducted with greater efficiency.

\section{EPV098/\#177 PROSPECTS FOR IMPROVING THE METHODS OF COMPLEX TREATMENT OF PATIENTS WITH ENDOMETRIAL CANCER STAGE I}

O Movchan*, V Svintsitskiy, O Renkas. National Cancer Institute, Oncogynecology, Kiyv, Ukraine

\subsection{6/ijgc-2021-IGCS. 168}

Objectives The analysis was performed in 968 women with endometrioid stage I endometrial cancer who underwent hysterectomy without/with adjuvant therapy (radiation or chemotherapy) in the Oncologynecology Research Department of the 


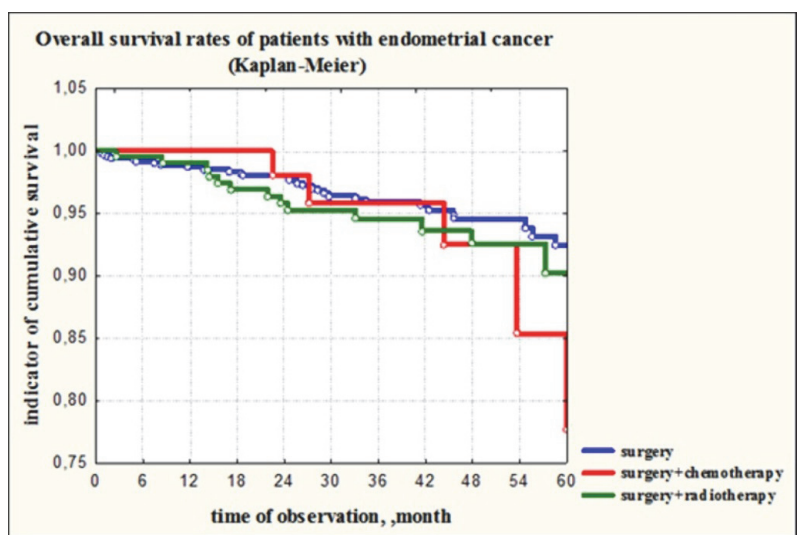

Abstract EPV098/\#177 Figure 1 Comparison of survival depending on the method of treatment in different groups of patients

National Cancer Institute from 2015 to 2020. Although threequarters received adjuvant treatment, recurrences occurred on average during the first three years.

Methods To evaluate the survival of patients with endometrioid stage I endometrial cancer depending on the type of treatment or their combination. The following statistical methods were used: standard descriptive and parametric. Survival of patients was analyzed by Kaplan-Meyer method. P values of $<0.05$ were considered significant.

Results Overall recurrence-free survival was $92.58 \pm 7.38 \%$ with a median non-recurrence survival of $34.3 \pm 14.7$ months. A total of 68 relapses were detected - 7.02\%. The median time from hysterectomy to the first recurrence, local and regional, was 6-18 months, respectively, and 24-36 months after combination treatment. The best survival was in the group of patients who received both surgical and chemotherapeutic treatment - averages of 59.5 months, and the worst after surgery - an average of 26.8 months ( $\mathrm{X}^{2}=1,031417$, $\mathrm{p}=0,59708)$ (See figure 1).

Conclusions Hysterectomy shows the most common recurrences of loco-regional, and the combination of surgical treatment with radiation therapy - increases the frequency of distant metastases. Surgical treatment with radiation or chemotherapy leads to improve recurrence-free survival.

\section{EPV099/\#179 ROBOTIC-ASSISTED SURGERY FOR ENDOMETRIAL CANCER IN MORBIDLY AND EXTREMELY MORBIDLY OBESE PATIENTS}

C Lechartier*, J Bernard, MC Renaud, M Plante. Hotel Dieu de Quebec, Gynecology Oncology, Quebec, Canada

\subsection{6/ijgc-2021-IGCS.169}

Objectives We sought to evaluate the outcome of roboticassisted surgery for endometrial cancer in morbidly obese (MO) and extremely morbidly obese (EMO) patients.

Methods We retrospectively reviewed all robotic gynecologic oncologic surgeries performed for endometrial cancer, in women with a BMI $\geq 40 \mathrm{~kg} / \mathrm{m} 2$, from 2012 to 2017 in our center. Patients were divided into two groups (MO: $40-49 \mathrm{~kg} /$ $\mathrm{m} 2$, EMO: $\geq 50 \mathrm{~kg} / \mathrm{m} 2$ ). Complications and outcome were compared. Fisher's test, t-test and Kaplan-Meier were used for statistical analyses.

Results Eighty-seven women were included: 64 (74\%) MO and 23 (26\%) EMO. The main histology was endometrioid adenocarcinoma (77\% of MO and $61 \%$ of EMO) and endometrial intraepithelial neoplasia (19\% of $\mathrm{MO}$ and $35 \%$ of EMO). The median blood loss was $100 \mathrm{~mL}$ in $\mathrm{MO}$ and $75 \mathrm{ml}$ in EMO $(p=N S)$. The median length of stay was one day for each group (range: 0-11). Two EMO (9\%) and none of the MO patients required conversion to laparotomy due to poor surgical field exposure $(p=0,067)$. Overall, 5 MO patients (8\%) and 5 EMO (22\%) had a surgical complication $(p=0,12)$, but only 3 patients ( $1 \mathrm{MO}$ and $2 \mathrm{EMO})$ required re-hospitalization within 30 days. The median follow-up was 47,7 months (range: 1,43-93,6). Recurrence occurred in $9 \%$ in each group, with no difference in recurrence-free survival $(p=0,96)$. Only one MO patient died of cancer recurrence.

Conclusions The robotic-assisted surgery for endometrial cancer in morbidly obese patients is a safe and feasible procedure. The morbidly obese and extreme morbidly obese patients appear to have similar oncologic outcome, length of hospital stay, blood loss and low surgical complications.

\section{EPV100/\#181 UTERINE CARCINOSARCOMA: A MULTICENTRE REVIEW OF TREATMENT AND OUTCOMES OVER 26 YEARS}

${ }^{1} \mathrm{C}$ Yim*, ${ }^{2} \mathrm{~S}-\mathrm{E}$ Yao, ${ }^{3}$ J Phung, ${ }^{4} \mathrm{M}$ Davies-Tuck, ${ }^{2} \mathrm{~T}$ Manolitsas, ${ }^{2} \mathrm{~J}$ Mcneilage, ${ }^{2} \mathrm{~K}$ Reid, ${ }^{5} \mathrm{O}$ Mcnally, ${ }^{6} \mathrm{R}$ Rome, ${ }^{2} \mathrm{~T}$ Jobling. ${ }^{1}$ Royal Women's Hospital, Gynaecologic Cancer Centre, Randwick, Australia; 'Monash Health, Gynaeoncology Unit, Moorabbin, Australia; ${ }^{3}$ University of Newcastle, School of Medicine and Public Health, Newcastle University, Australia; ${ }^{4}$ Hudson Institute of Medical Research, Epidemiology and Clinical Trials, Clayton, Australia; ${ }^{5}$ Royal Women's Hospital, Gynaeoncology Unit, Parkville, Australia; ${ }^{6}$ Epworth Healthcare, Epworth Freemasons Hospital, East Melbourne, Australia

\subsection{6/ijgc-2021-IGCS.170}

Objectives Uterine carcinosarcoma (UCS) is a rare neoplasm with a poor prognosis and a paucity of evidence on treatment. The objective was to review the characteristics, treatment and outcomes of UCS cases across two gynaeoncology units and five private gynaeoncology practices in Melbourne.

Methods UCS cases were identified from hysterectomy pathology records between 1994 and 2020 inclusive. Patient characteristics, histopathological stage, adjuvant therapy, recurrence and survival status were extracted from patient records.

Results 208 cases of UCS were identified. The overall recurrence rate was $26.0 \%$ and the overall death rate was $60.1 \%$. Increasing age at diagnosis was associated with an increased risk of death (adjusted OR 1.04, 95\% CI 1.01-1.08, $\mathrm{p}=0.019$ ). Risk of death was highest in Stage III disease (adjusted OR 4.37, 95\% CI 1.67-11.40). Recurrence was a strong determinant of death, with an adjusted OR of 7.58 $(\mathrm{p}<0.001)$.

Conclusions In this relatively large homogenous cohort of UCS cases, significant predictors for survival included age at diagnosis, stage of disease and recurrence. 